\title{
Self-Organization of Surface Transportation Networks
}

\author{
David Levinson \\ Department of Civil Engineering, University of Minnesota, \\ 500 Pillsbury Drive Southeast, Minneapolis, Minnesota 55455, levin031@umn.edu \\ Bhanu Yerra \\ Thurston Regional Planning Council, 2424 Court, Southwest, Suite A, Olympia, Washington 98502, \\ yerrab@trpc.org
}

\begin{abstract}
$T^{1}$ his research investigates the self-organization of surface transportation networks. Using a travel demand model coupled with revenue, cost, and investment models, experiments are run under a variety of parameters on a grid network. It is found that roads, contiguous sections of multiple links operating with similar characteristics, and hierarchies of roads emerge under a broad range of assumptions from networks with neither defined roads nor clearly organized hierarchies. The factors that drive this are the (dis)economies of scale, the presence of boundaries, and any initial asymmetry in the network. This research thus finds that roads and hierarchies, which are often thought to be the product of conscious design, can also arise without such intention.
\end{abstract}

Key words: network evaluations; self-organization; network growth

History: Received: February 2005; revision received: June 2005; accepted: August 2005.

\section{Introduction}

Casual observation suggests that transportation networks exhibit organization. There is a hierarchy of roads, so that some roads are more important (wider, faster, carry more traffic) than others. Moreover, the fact that there are roads, and not just random links, wherein the characteristics of links are often congruent (have similar flow and capacity characteristics) with the characteristics of some upstream and downstream links, is another indication of organization. This phenomenon could be the result of design and planning. This paper examines how this phenomenon may result from the complex interaction of independent links, treating links as autonomous agents. Thus, transportation network properties such as the existence of roads, and a hierarchy of roads, can be the result of self-organization.

The formation of hierarchy of roads within networks is posited to be independent of the hierarchy of places. However, there is much to learn from study of central place theory developed by Christaller (1933) and Lösch (1954). Christaller presents three principles (market, traffic, and sociopolitical) that govern the formation of central places. The market principle states that central places emerge to maximize each one's market area subject to transportation costs, market areas that Lösch suggests take a hexagonal shape. The traffic principle states that central places emerge to maximize the satisfied demands of transportation while minimizing the cost of transportation and infrastructure provision. Although Christaller lists the underlying principles that govern the formation of transportation network, he does not explain the formation of hierarchies of roads, as his subject is central places, not networks. Krugman (1996) criticizes these models as being noneconomic because they do not show emergence of these central places from decentralized processes. Recently, Fujita, Krugman, and Venables (1999) formulated a mathematical model that explains the hierarchical formation of cities from a "decentralized market process." This research aims to fill the lacuna by modeling transportation networks, using a decentralized process, to observe the conditions under which the hierarchies of roads emerge.

Researchers have studied the growth and change of the physical infrastructure network from a microscopic perspective. Taaffe, Morrill, and Gould (1963) examine undeveloped countries to suggest a model for the growth of the transportation network; Garrison and Marble (1965) explain the order of rail network construction in Ireland by showing that nodes would connect to the nearest large neighbor. Schweitzer and Schimansky-Geier (1994), Lam and Pochy (1993), and Lam (1995) introduce an activewalker model (AWM) in which walkers moving on a landscape change it, a model adopted by Helbing, Keltsch, and Molnár (1997). Watts and Strogatz (1998), Barabasi and Albert (1999), and Barabasi, Albert, and Jeong (1999) propose models that explain the properties and evolution of networks and measure the formation of hierarchies of node connectivity, defined 
for a node as the number of adjacent nodes that are one link away, obtaining a power law distribution (see Bak 1996). However, because road junctions are typically limited in the number of connections allowed (most nodes will connect three or four road segments, five or more create significant traffic issues), link connectivity is the more pertinent issue in surface transportation networks. Research into transportation networks can greatly benefit from Barabasi, Albert, and Jeong's (1999) concept of preferential attachment, which is akin to the concept of the "rich get richer," as the source of hierarchies. Yamins, Rasmussen, and Fogel (2003) model road growth co-evolving with urban settlements from an empty space with highly simplified travel demand and road supply mechanisms. Levinson and Karamalaputi (2003a, b) estimate empirical models explaining the expansion of links and the construction of new links.

It is intuitive that hierarchical properties of roads emerge if central places preexist. That may be putting the cart before the horse because many of those central places exist because of the intersection of transportation network elements. To understand network organizational properties, this paper assumes there are no central places; land uses and population are distributed uniformly. While land use and transportation networks are mutually dependent systems in the sense that change in one affects the growth (or decay) of the other, this research neglects the dynamics that involve the effects of the network on land use in order to understand network properties in isolation.

An agent-based model is developed to model the organization, growth, and contraction of transportation network elements. The components model travel demand, revenue, cost, and investment. The travel demand model converts this population and employment data into traffic using the given network topology and determines the link flows by following the traditional planning steps of trip generation, trip distribution, and traffic assignment (for simplicity, a single mode is assumed). A revenue model determines the price the traffic must pay for using the road depending on speed, flow, and length of the link. A cost model calculates the cost required to maintain link speeds depending on traffic flow. Revenue in excess of maintenance costs will be invested on the link to improve its condition using an investment model until all revenue is consumed. After upgrading (or downgrading) each link in the network, the time period is incremented and the whole process is repeated until an equilibrium is reached or it is clear that it cannot be achieved. An overview and interconnection of these models is shown in Figure 1.

Using this model a variety of network topologies are tested to study the properties (in particular the internal hierarchy of roads) of transportation

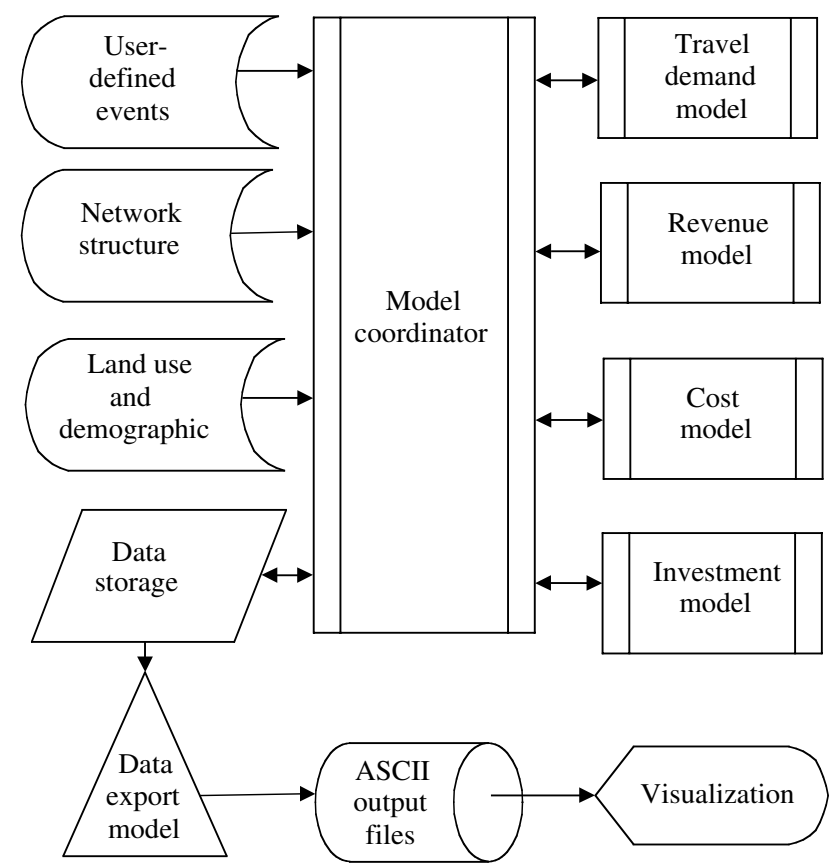

Figure 1 An Overview of the Model

networks. A grid network is used as a base case because of its prevalence and its ease of maintaining uniform distribution of link lengths and area of traffic zones. Cylindrical and torus network topologies are also employed in order to test the effect of finite no-boundary topologies and compare the results with finite planar topologies, which will help in understanding the intrinsic properties of planar networks. In particular, we want to understand how boundaries affect the results. We investigate the nature of self-organization of the network. Self-organization is a process in which a system's order increases without outside direction, and is sometimes referred to as emergence. We apply that within the fixed spatial structure of road networks to observe the formation of a hierarchy of roads within a given lattice.

The model employed in this paper is presented in the next section. The experiments are described in the following section, along with results. A measure of congruence is developed and used to demonstrate the degree of self-organization. The final section presents the conclusions and the potential consequences of this research on transportation planning and policy.

\section{Model}

The transportation network is modeled as a directed graph overlaying a land-use layer comprising a grid of land blocks called land-use cells. The network layer and land-use layer are connected to exchange information between layers. The model is intentionally simplified to convey the results most clearly with a minimum of confounding factors. 


\section{Network}

Planar Network. Local roads in many cities possess a grid structure. Their prevalence and simplicity are not the only reasons why they are considered: The uniformity in link length, trips produced, and trips attracted is easy to control in grid networks. Because the main objective is to show whether roads and hierarchies in transportation networks are an emergent property, it is important to maintain uniformity, and a grid network provides the required flexibility. To facilitate comparison in this paper, grids with 225 nodes arranged in a square (15 network nodes running longitudinally by 15 nodes laterally) are considered. The authors have tested other size networks and found very similar properties (although slightly different for networks that have an even number of longitudinal or latitudinal nodes than for networks with odd numbers due to symmetry properties), which are excluded here for brevity. The main drawback of using a grid network is that the effect of edges on the formation of hierarchies cannot be eliminated. In order to eliminate edge effects, two additional cases involving are considered.

Cylindrical Surface. A grid network on a cylindrical topology, as shown in Figure 2, although unrealistic, eliminates edge effects in one direction. However, edges that are parallel to the brims of the cylinder may still affect the hierarchies.

Torus Surface. A grid network on a torus surface is considered to eliminate the discontinuities associated with a grid network on planar and cylindrical surfaces. However, on a torus surface a grid network will result in nonuniformly distributed link lengths. In order to overcome this, nodes along opposite sides of a grid network are forcibly connected in such a way that link lengths are uniformly distributed through out the resulting network. In such a torus world, theoretically, it can be argued that every link

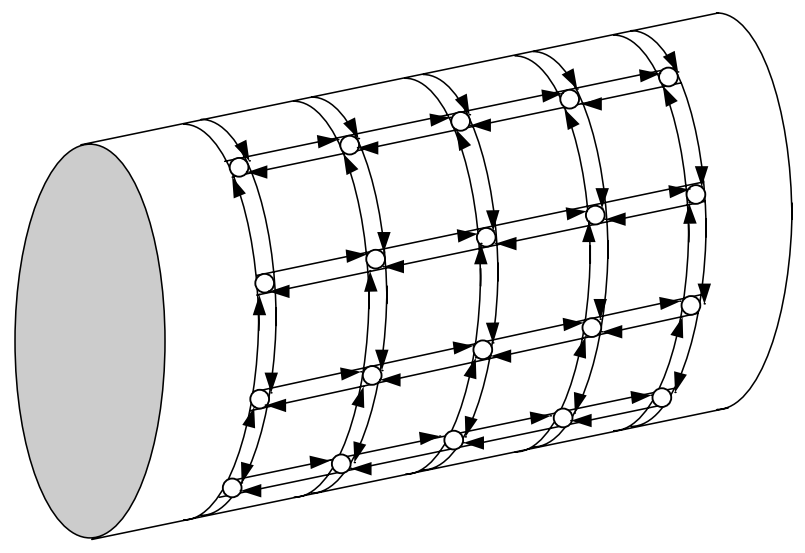

Figure 2 A Grid Network on Cylindrical Surface is indistinguishable from every other link irrespective of its orientation, provided that land use and travel behavior are uniform.

\section{Travel Demand Model}

The travel demand model converts the given land use into link flows. At any point in time, the flow on a link results from many individuals' decisions. Individuals decide how often to travel, where to travel, what mode of transportation to choose, and which route to take. Transportation engineers and planners often model these decisions, respectively, using Trip Generation, Trip Distribution, Mode Choice, and Traffic Assignment. To simplify the analysis, a single vehicle type is considered, thereby eliminating mode choice.

Trip Generation. Trip generation converts the land-use properties into trips produced and attracted from a zone. The model allocates land-use cells to the nearest network node $\left\{O_{n}\right\}$. Because the terms zone and network node convey the same idea in this model, they are used interchangeably. The trip generation model calculates the total trips produced $p_{r}$ and attracted $q_{s}$ from a network node by summing the trips from land-use cells in $\left\{O_{n}\right\}$.

Trip Distribution. Trip distribution sends trips from origins to destinations. This step generates an origin-destination matrix (OD matrix), with its elements representing the number of trips generated from an origin zone or origin network node, given by the elements row number, to a destination zone or destination network node, given by the elements column number. Because every zone is both an origin and destination, and the trips originating and ending in the same zone are not counted; the OD matrix is a square matrix with zeros along its diagonal. Let $m_{r s}^{t}$ represent elements in the OD matrix, which means $m_{r s}^{t}$ is the number of trips generated from network node $r$ and ending at network node $s$ in time step $t$. Then the following relationships hold at every time step $t$.

$$
\begin{aligned}
& p_{r}=\sum_{s=1}^{N} m_{r s}^{t} \quad \forall r \in\{N\} \\
& q_{s}=\sum_{r=1}^{N} m_{r s}^{t} \quad \forall s \in\{N\}
\end{aligned}
$$

Note that $r$ represents an origin network node and $s$ represents a destination network node. The singly constrained gravity model is adopted in this research (Hutchinson 1974).

To define the generalized cost of commuting between any two nodes, it is necessary to define the generalized cost of commuting on a link. The generalized cost of commuting on a link $a$ in time step $t$ is 
assumed to consist of two parts; travel time converted to monetary value and toll.

$$
d_{a}^{t}=\frac{l_{a}}{v_{a}^{t}}+\rho_{0}\left(l_{a}\right)^{\rho_{1}}\left(v_{a}^{t}\right)^{\rho_{3}}
$$

where

$d_{a}^{t}$ is the generalized cost of commuting on arc $a$

$l_{a}$ is the link length

$v_{a}$ is the link speed

$\rho_{0}, \rho_{1}$, and $\rho_{3}$ are coefficients.

One way of calculating generalized cost between any origin and destination nodes is by summing up generalized cost of all the links along the preferred path between the nodes. Because the routes that travelers take are not known at this stage, the ambiguity one faces is how to calculate the $d_{r s}^{t}$ between any given $r$ and $s$. Two assumptions are made at this stage. First, congestion or the feedback effect between travel time and flow (link performance function) is neglected. Second, all trips between any origin and destination follow the same route, which is the shortest path between the nodes. The algorithm used to calculate the shortest path is presented in the next section. Let $\left\{K_{r s}^{t}\right\}$ represent a set of links that are along the shortest path between the origin node $r$ and destination node $s$. Using these two assumptions, the generalized cost of travel between any two nodes is calculated using:

$$
d_{r s}^{t}=\sum_{a \in A} d_{a}^{t} \delta_{a, r s}^{t} \quad \forall r, s \in\{N\}
$$

where

$\delta_{a, r s}^{i}$ is a dummy variable equal to 1 if link $a$ belongs to $\left\{K_{r s}^{t}\right\}, 0$ otherwise.

$d_{r s}^{t}$ is the generalized cost of commuting between nodes $r$ and $s$.

The number of trips in each OD pair is

$$
m_{r s}^{t}=p_{r} \frac{q_{s} \cdot h\left(d_{r s}^{t}\right)}{\sum_{s} q_{s} \cdot h\left(d_{r s}^{t}\right)} \quad \forall r, s \in\{N\}
$$

where the negative exponential model is used:

$$
h\left(d_{r s}\right)=e^{-w \cdot d_{r s}} .
$$

Traffic Assignment. Traffic assignment uses an allor-nothing shortest-path assignment based on the link costs. Dijkstra's algorithm (Chachra, Ghare, and Moore 1979) is used to calculate the shortest path. The drawback of using Dijkstra's algorithm is that it does not enumerate all the possible shortest paths, if there is more than one. When there are multiple equal travel time paths between an origin and destination, Dijkstra's algorithm chooses one based on the arbitrary numbering of nodes. This is a disadvantage especially when a network is either symmetric or can be mapped onto itself by permuting the node numbers. Using symmetric conditions in solving traffic assignment problems is not new to transportation planning; it was suggested by Newell (1980) "if traffic is assigned to routes so as to minimize some convex function of the link flows, then the resulting link flows must show the same symmetry as the network itself." Therefore, in cases where land use and network are symmetric, link flows are averaged with their images from all possible symmetric axes. In the case of a grid network there are four possible symmetric axesvertical axis, horizontal axis, and two diagonal axes. The combinations of these axes produce eight distinct images of any link, including the original link. Flows from Dijkstra's algorithm for all these eight links are averaged to obtain the resulting flow on the links. This is performed on every link of the network to obtain the symmetric flows. This is one modeling assumption among many that could be made. Alternatives include using logit (or other discrete-choice) models for path spreading. These alternatives may produce different assignment results (as on a perfectly symmetric network there are many equal-time paths between distant origins and destinations), which may change the specific outcomes, but we believe will not change the fundamental results.

\section{Revenue Model}

The model calculates revenue for each link by multiplying the toll and flow. Therefore, the higher the flow on the link, the higher is the revenue $\left(E_{a}\right)$. The toll is approximately equivalent to a gas tax if it is proportional only to link length when the central gastax-collecting authority allocates revenue back to links proportional to the amount of gas tax revenue those links generate (the idea behind shadow tolls).

$$
E_{a}^{t}=\left(\rho_{0} \cdot\left(l_{a}\right)^{\rho_{1}}\left(v_{a}^{t}\right)^{\rho_{3}}\right) \cdot\left(\psi \cdot f_{a}^{t}\right) \quad \forall a \in\{A\}
$$

where

$\psi$ is a model parameter to scale flow $\left(f_{a}\right)$ annually, and

$$
\rho_{0}, \rho_{1}, \rho_{3} \text { are coefficients. }
$$

\section{Cost Model}

This model calculates the cost to keep a link in its present usable condition depending on the flow, speed, and length. A Cobb-Douglas model is assumed as shown below.

$$
C_{a}^{t}=\mu \cdot\left(l_{a}\right)^{\alpha_{1}}\left(f_{a}^{t}\right)^{\alpha_{2}}\left(v_{a}^{t}\right)^{\alpha_{3}} \quad \forall a \in\{A\}
$$

where, $C_{a}^{t}$ is the cost of maintaining the road at its present condition in time step $t$,

$\mu$ is the (annual) unit cost of maintenance for a link, $\alpha_{1}, \alpha_{2}, \alpha_{3}$ are coefficients indicating economies or diseconomies of scale.

The cost calculated using this model depicts a threshold cost of maintaining a link. 


\section{Investment Model}

Depending on the available revenue and maintenance costs, this model changes the speed of every link at the end of each time step as shown in Equation (9). If the revenue generated by a link is insufficient to meet its maintenance requirements, i.e., $E_{a}<C_{a}$, its speed drops. If the link has revenue remaining after maintenance, it invests that remaining amount in capital improvements, increasing its speed. This, along with a shortest-path algorithm, embeds the "rich get richer" logic of link expansion. A major assumption in this model is that a link uses all the available revenue in a time step without saving for the next time step.

$$
v_{a}^{t+1}=v_{a}^{t}\left(\frac{E_{a}^{t}}{C_{a}^{t}}\right)^{\beta} \quad \forall a \in\{A\}
$$

where $\beta$ is speed improvement coefficient.

With the new speed on the links the travel time, and consequently shortest path, changes; the whole process from the travel demand model is iterated to grow the transportation network until the network reaches equilibrium, or it is clear that it will not.

\section{Averaging Link Flows}

In reality, the investment on roads is such that the money spent upgrading a link from $m \rightarrow n$ is generally the same as investment upgrading a link from $n \rightarrow m$. However, the link speeds from the model do not necessarily comply, especially when initial speeds are randomly distributed, and symmetry conditions in traffic assignment cannot be applied. In order to solve this, the speeds on adjacent links are averaged at the end of the investment model. This is, again, one modeling assumption, and alternative assumptions would produce different specific results.

\section{Experiments and Results}

The network dynamics model presented above provides a platform to conduct experiments on transportation networks to study their properties and growth dynamics. Several experiments are conducted, and the results are presented in this section. These experiments release constraints in the base case, one at a time, and explain the significance of the results. Some of the experiments in this section are considered to investigate the effect of the coefficients on the base case.

\section{Base Case}

A base case is chosen, and variations are made to this case to study how these variations affect the dynamics and the resulting hierarchy of roads. The base case consists of an evenly spaced grid network in the form of a square, with each link having the same speed. Each land-use cell produces and attracts the same
Table 1 Model Parameters and Values Used for Experiments

\begin{tabular}{llc}
\hline Variable & \multicolumn{1}{c}{ Description } & Base assumption \\
\hline$v_{a}^{0}$ & Initial speed (integer) & 1 \\
$g_{z}, h_{z}$ & Land-use properties of cell $z$ & 10 \\
$w$ & Coefficient in trip distribution model & 0.01 \\
$\rho_{0}$ & Coefficient in revenue model & 1.0 \\
$\rho_{1}$ & Length power in revenue model & 1.0 \\
$\rho_{3}$ & Speed power in revenue model & 0.0 \\
$\tau$ & Tax rate in revenue model & 1.0 \\
$\psi$ & Revenue model parameter & 365 \\
$\mu$ & Unit cost in cost model & 365 \\
$\alpha_{1}$ & Length power in cost model & 1.0 \\
$\alpha_{2}$ & Flow power in cost model & 0.75 \\
$\alpha_{3}$ & Speed power in cost model & 0.75 \\
$\beta$ & Coefficient in investment model & 1.0 \\
\hline
\end{tabular}

number of trips because of uniformly distributed population and employment. The network structure and land-use properties are chosen this way to eliminate network asymmetries as a confounding factor. Speeds on links running in the opposite direction between the same nodes are averaged in this case. Because Dijkstra's algorithm does not list all possible shortest paths between any two nodes, symmetry conditions are externally applied. Table 1 shows the parameters used in the base case and other experiments.

Figure 3 shows the spatial distribution of speed for the network at equilibrium. The entire range of link speeds is divided into four equal intervals, and these interval categories are used in drawing the figure with the line thickness and color representing the speed category. The spatial distribution of speeds depends on the parameters used in the model. Results clearly show that hierarchies and roads emerge from a localized link-based investment process. We believe one factor driving the emergence of the hierarchy of roads is the travel behavior induced by the presence of a boundary. Travel demand along the edges is inward, while trips are evenly distributed outward in all possible directions from the center. We explore this in a later section.

\section{Economies of Scale}

Having shown the formation of road hierarchies, it is now time to reason for the converging solution. For a given static land use, the economies of scale in the cost function associated with traffic flow $\left(\alpha_{2}<1\right)$ along with the increasing cost for higher link speeds $\left(\alpha_{3}>0\right)$ drives the system to an equilibrium. Diseconomies of scale in the cost function with respect to traffic flows, while maintaining $\alpha_{3}$, result in an oscillating equilibrium.

The values of coefficients in the cost model not only signify the economies of scales, but also help in bringing the system to an equilibrium. This experiment 

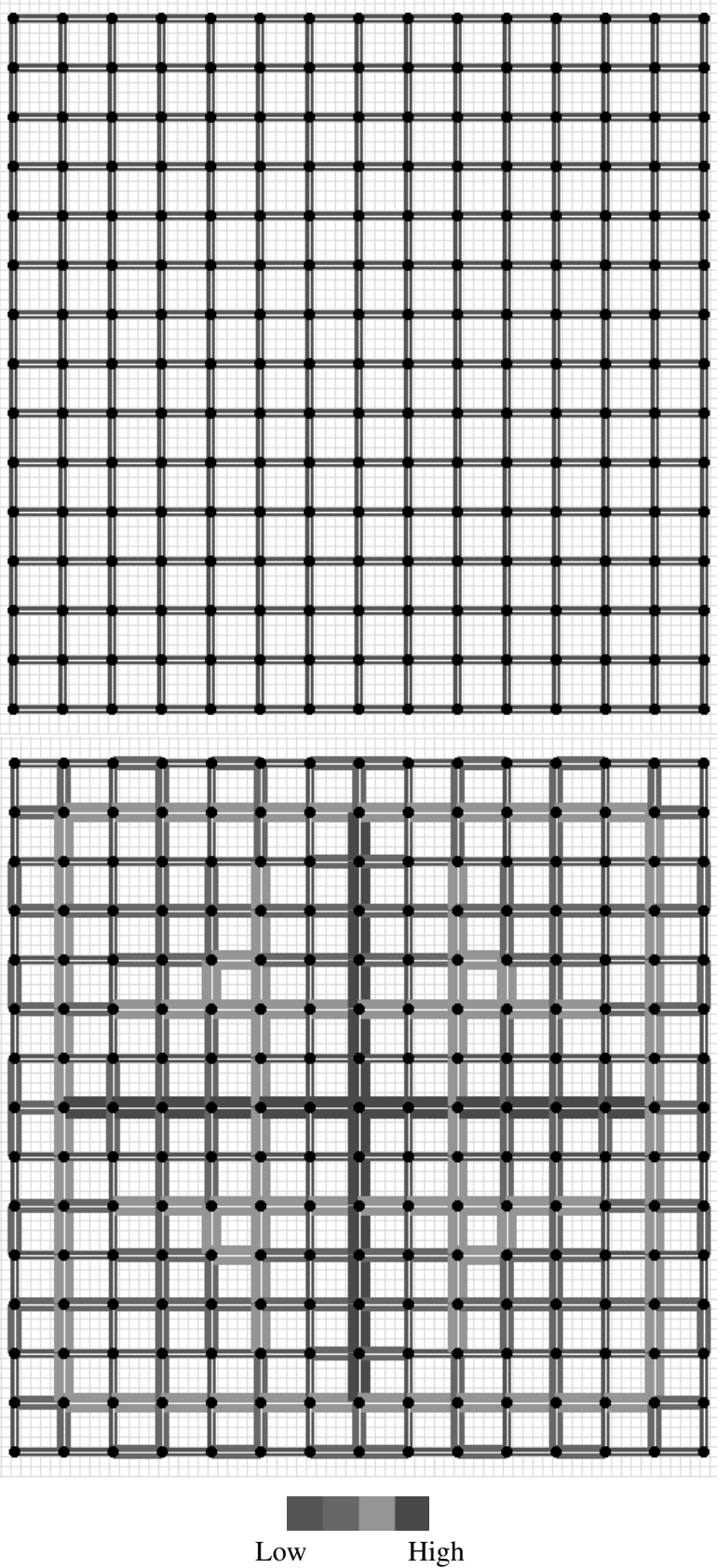

Figure 3 Base Case: Uniform Initial Speeds

Notes. Top: spatial distribution of speed for the initial network.

Bottom: spatial distribution of speed for the network at equilibrium reached after eight iterations.

A hierarchy of roads emerges without any initial differentiation of speeds.

considers the possible range of these coefficients and investigates their effect on the equilibrium. The range of each coefficient is divided into three intervals; less than zero, between zero and one, and greater than one. The resulting nine spaces and the outcome of the network dynamics in each space are shown in Table 2. Only in a few spaces does the system reach an equilibrium, and in others the system oscillates or diverges.
Table 2 Equilibrium Average System Speed for Different Values of $\alpha_{2}$ and $\alpha_{3}$ in Cost Model Either Oscillates or Diverges to Infinity or Converges to a Very Small Value Close to Zero

\begin{tabular}{lccc}
\hline & \multicolumn{3}{c}{ Coefficient on flow } \\
\cline { 2 - 4 } Coefficient on speed & $\alpha_{2}<0$ & $0<\alpha_{2}<1$ & $\alpha_{2}>1$ \\
\hline$\alpha_{3}>1$ & Infinity & Equilibrium & Oscillates \\
$0<\alpha_{3}<1$ & Infinity & Equilibrium & Oscillates \\
$\alpha_{3}<0$ & Infinity & Infinity & Near zero \\
\hline
\end{tabular}

These results make some intuitive sense. First the left column: If total cost decreases as flow increases, it is unsurprising that we obtain infinite speeds. Similarly, the bottom row shows that when total cost decreases as speed increases, we get infinite speeds, at least until offset by diseconomies of flow. Oscillations in speeds and flows are seen when diseconomies of scale in the cost function with respect to flow exist, provided the cost increases with speed.

\section{Random Initial Link Speeds}

This experiment is similar to the base case except for randomly distributing the initial link speeds between 1 and 5 . Because speeds are randomly distributed, symmetric conditions in traffic assignment are not applied, and the investment model averages speeds on adjacent links. The network is evolved until an equilibrium is reached. Because random distribution of initial link speeds makes the results stochastic, 20 such simulations were performed. A typical solution is shown in Figure 4.

Emergence of hierarchies and roads are clearly seen in this case. Random distribution of initial speeds produces nonsymmetrically oriented roads with most of the faster links concentrated in the center. Belt or ring roads are common. Figure 4 shows the spatial distribution of traffic flow. Notice that there are fewer links that carry a high flow of traffic (thicker links in red) and many links that carry less traffic (thinner links in green), resembling Zipf's Law, a rank-order rule that has been applied to city sizes, wherein the frequency of the $n$th largest city is inversely proportional $n$.

\section{Cylinder and Torus Networks}

Results suggest that one reason the hierarchy of roads forms is due to the presence of boundaries; in order to examine topologies without boundaries, cylindrical and torus surfaces are considered in this experiment. Although these topologies are strictly theoretical in the context of surface transportation, they are not strange in the communication and distributed computing fields. Except for the topology, this experiment does not differ from the case with random initial speeds. Because the initial speeds are random, 20 such cases are performed. Results for a typical cylindrical case are shown in Figure 5. These figures are drawn 

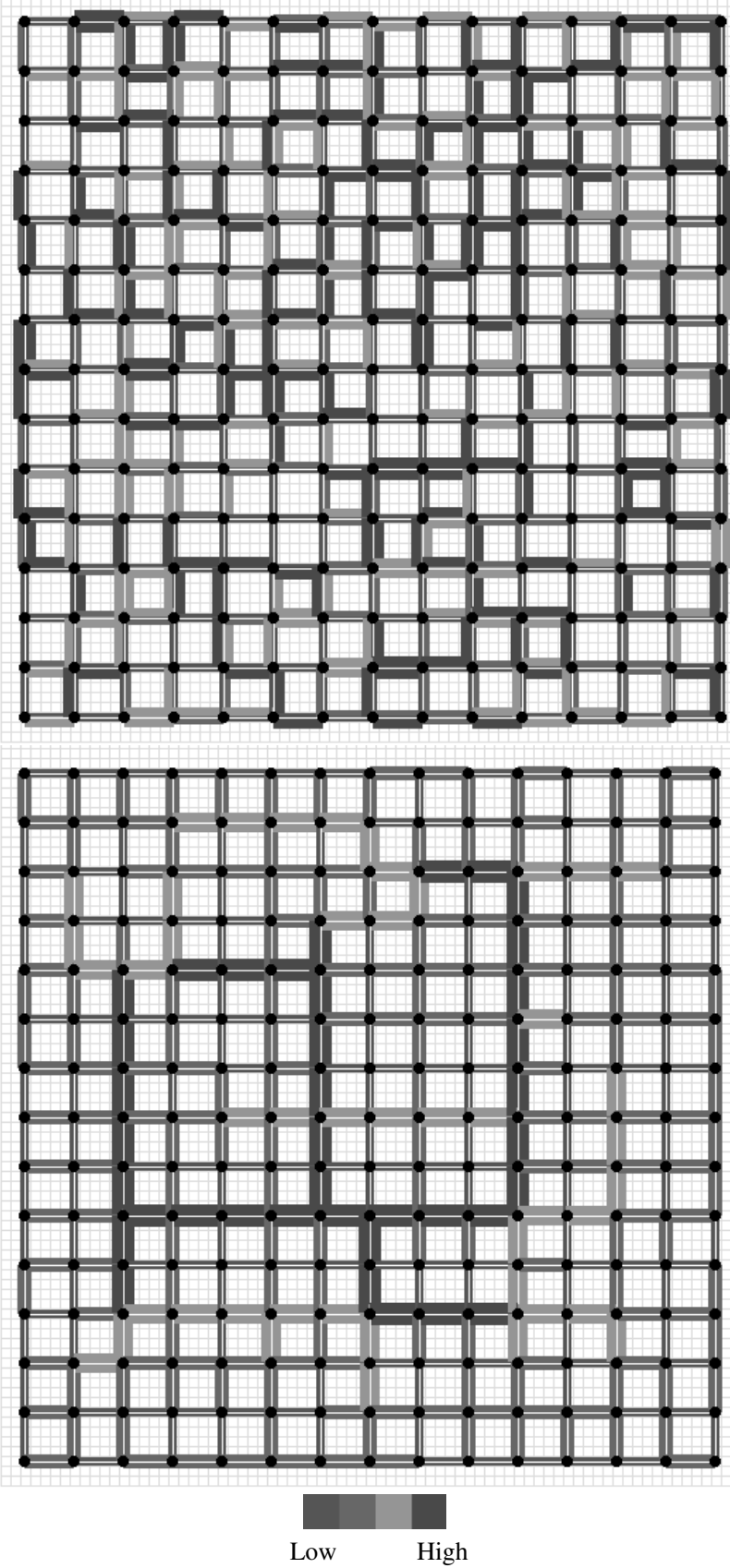

Figure 4 Random Initial Speeds

Notes. Top: spatial distribution of initial speeds.

Bottom: spatial distribution of speeds for the network at equilibrium.

A random initial distribution of speeds will still form a hierarchy of roads, but one that is not symmetric.

by cutting the surface of the cylinder parallel to its longitudinal axis and spreading the surface flat. Note that high-speed links are away from the boundarybehavior that was observed earlier. Formation of beltways and ring roads are again seen this case.

Equilibrium spatial distribution of speeds for the torus topology can be seen in Figure 6. These figures are drawn by cutting only one ring of the torus
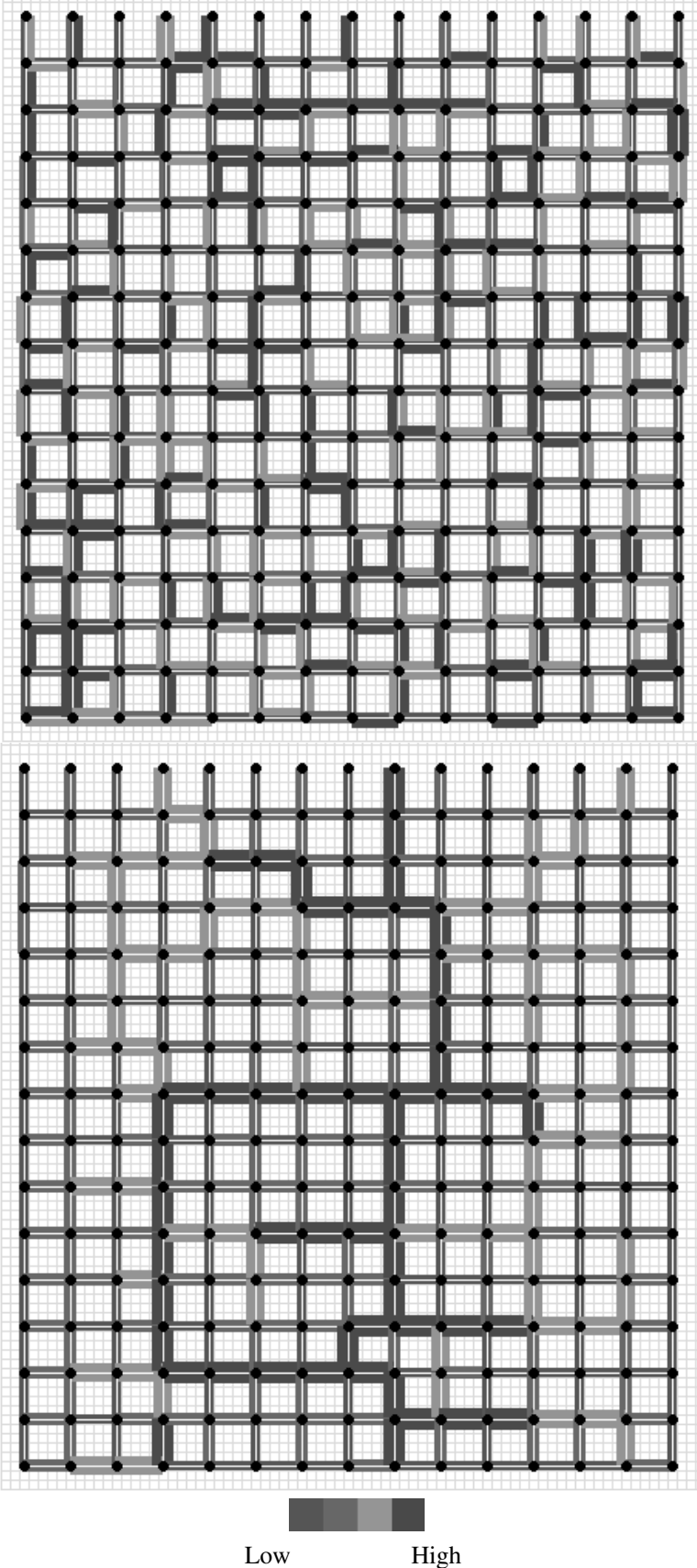

Figure $5 \quad$ Cylindrical Network

Notes. Top: spatial distribution of initial speed for a cylindrical topology. Bottom: spatial distribution of speeds for the network after reaching equilibrium.

The color and thickness of the linkshows its relative speed or flow. Results are similar to random initial speed on the grid network, although now there is one principal north-south axis, which loops around from the top of the figure to the bottom.

surface along a plane that passes through the longitudinal axis and then slicing the resulting cylindrical surface to spread it on a plane. Because there are no boundaries in this topology, the spatial distribution of high-speed links is not skewed. Beltways and ring roads formed in this topology too. 


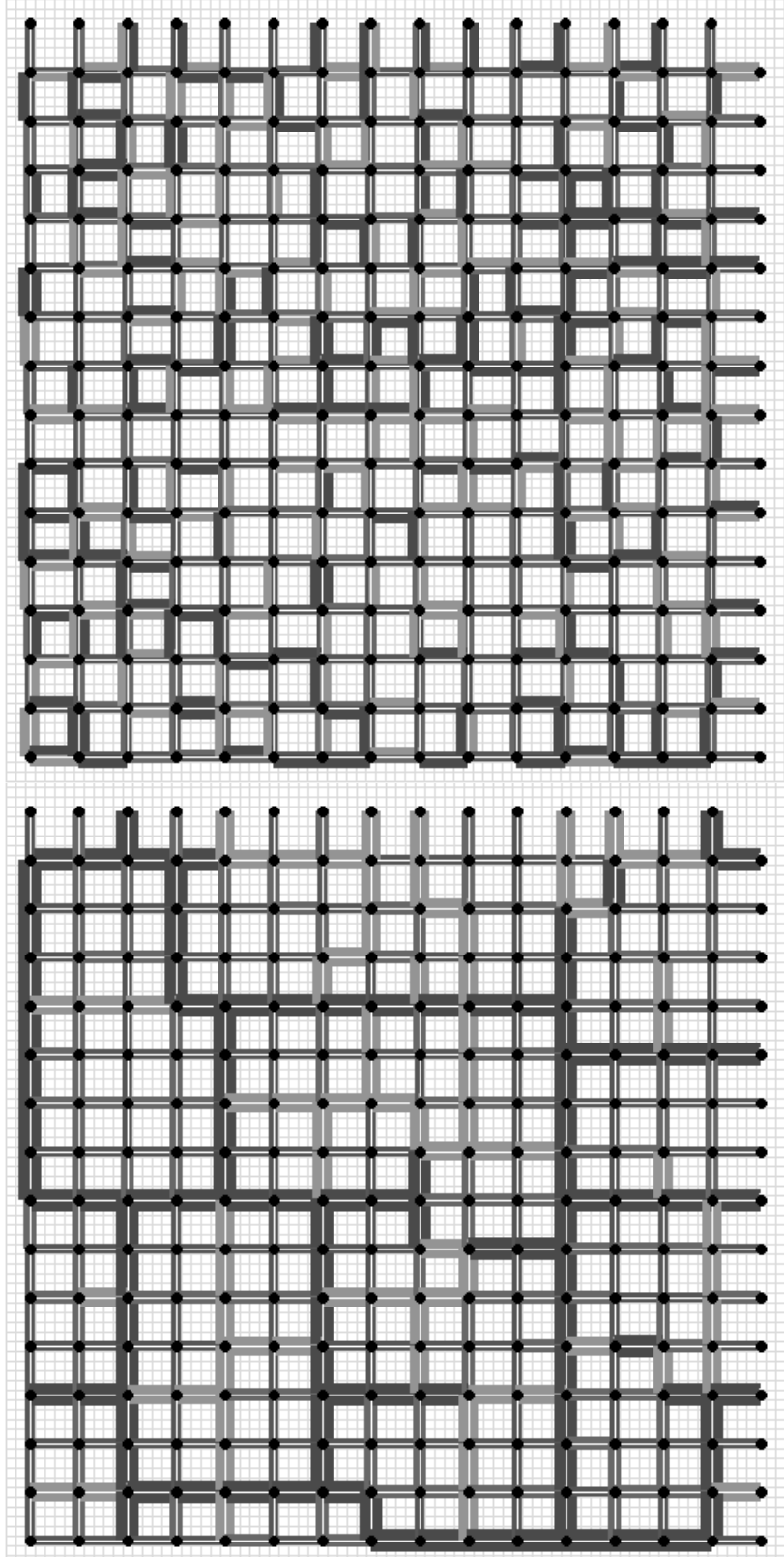

Figure 6 Torus Network

Notes. Top: spatial distribution of initial speed for torus topology. Bottom: spatial distribution of speeds for the network after reaching equilibrium.

The color and thickness of the link shows its relative speed or flow. While similar to a random initial speed, high-speed links now appear on the edge of the figure, which is not the edge of the graph.

\section{A River Runs Through It}

The network used in this experiment is as shown in Figure 7, with a river flowing along the diagonal. This is a grid network in which the links around the diagonal are removed and the resulting disconnected grid structures are connected by long bridges and a series of links parallel to the riverbank. Because there is a river along the diagonal, the trips produced
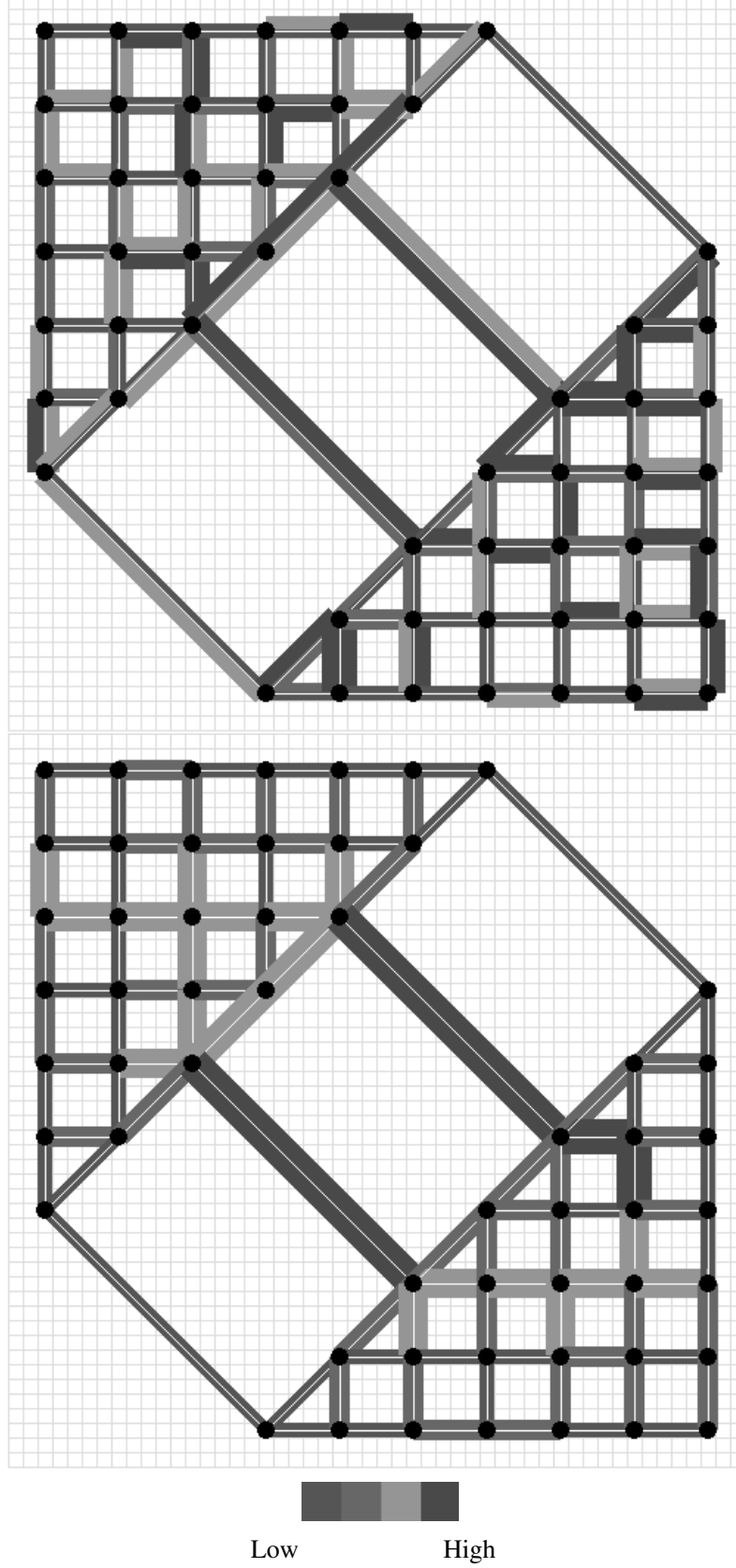

Figure $7 \quad$ River Network

Notes. Top: spatial distribution of initial speed for river network. Bottom: spatial distribution of final speeds.

Bridge links achieve special significance, and their feeder links get reinforced as well.

and attracted from these land-use cells are zero. Every other land-use cell produces and attracts 10 trips. Initial speeds are distributed between 1 and 5 , and a typical result is shown in Figure 7. The bridges in the center of the network are carrying more traffic, and the feeder links to these bridges are in the immediate next level of the hierarchy of roads. 


\section{Congruence}

There are a number of measures of organization that one could propose. Because we are interested in the hierarchy of roads, we are interested in how similar links are to upstream and downstream links. We define congruence $\left(c_{a}\right)$ for a link as the ratio of the minimal difference of speed between the examined link and its downstream (or upstream) links to the speed of examined link.

$$
c_{a}=\min _{i \in[1, K]}\left(\frac{\left|v_{a}-v_{b_{i}}\right|}{v_{a}}\right)
$$

where $v$ represents link speed, and $a$ and $b$ are the link in question and the upstream or downstream link, respectively, and $i$ is an index of all upstream or downstream links connected to the link of interest.

A congruence of 0 is completely congruent, and higher numbers are less congruent. The congruence measure of a network is defined as the average of link congruence measures across all links in the network. Figure 8 shows the evolution of network congruence by iteration from two starting points, corresponding to two of the above experiments: uniform and random initial speed (where the initial link speed is randomly distributed between 1 and 10). As can be seen, in the case of a uniform initial speed, the network becomes less congruent because link speeds no longer remain uniform. However, with a random initial speed, the network becomes more congruent, indicating an increase in organization. Still, the uniform initial speed network is more congruent than the random initial speed, so while order increases in the latter case, it does not become more ordered than a situation that began highly ordered. This provides some quantitative measures of self-organization.

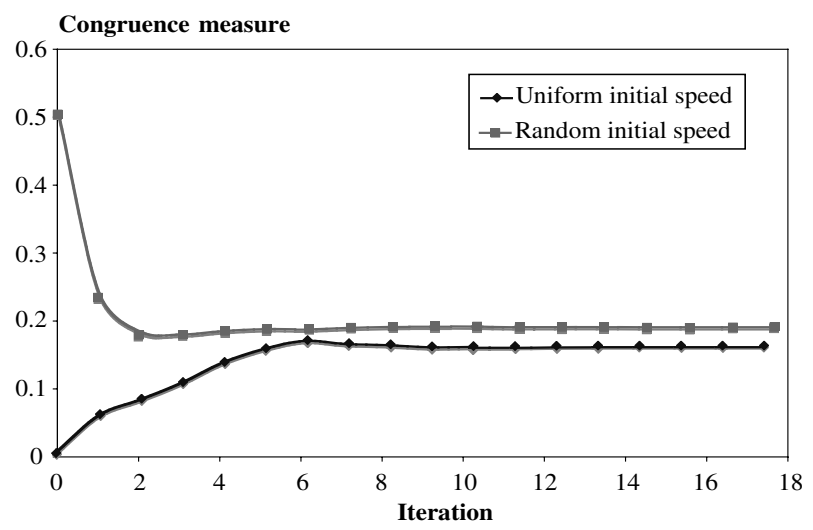

Figure 8 Measure of Congruence

Notes. Random initial speed network becomes more congruent (while uniform initial network becomes less congruent), although both converge to similar levels of congruence.

\section{Conclusions and Consequences}

Transportation network dynamics are inherently complex due to the numerous human decisions involved; not just the decisions of travelers, but the decisions of transportation financers (e.g., state departments of transportation), transportation policy makers, transportation modelers, and politicians. These individual and institutional decisions are the seemingly apparent causes for observed transportation network system properties like hierarchies, major roads, and congestion. This research models the emergence of hierarchies of roads and major routes by implicitly modeling the factors that affect decisions. The importance of this research is not just to theoretically demonstrate that the aforementioned transportation system properties are intrinsic, but also to emphasize the subtlety of human decisions in shaping the network.

The model adopted integrates the traditional travel demand model with link-based (or agent-based) revenue, cost and investment models. On the specified network structure, with uniformly or randomly distributed initial speeds and land use, the travel demand model calculates the link flows using a shortest-path algorithm, a toll is collected, then the revenue generated by traffic on that link is invested for the link's own growth. The cost model specifies the threshold of maintenance for each link, and if revenue generated by a link exceeds the cost, the investment model uses that revenue to increase the speed of the link, otherwise, the lack of maintenance erodes the speed of the link. This renewed network with new link speeds results in different travel behavior, and the whole process is iterated until an equilibrium in network speeds is reached or it is determined that there is no equilibrium.

The experiments and results give a sample of controlled network dynamics experiments that can be performed. Usually, consequences of such agentbased models are hard to predict although the dynamics are deterministic; such is the case here too. The base case demonstrates that despite maintaining uniform land use, initial speeds, link length, and node connectivity (except for the network nodes along the edges, nodes are adjacent to exactly four other nodes), self-organizing hierarchies arise due to the presence of boundaries and economies of scale. When initial speeds are randomly distributed, the link speeds and flows self-organize to form roads that are more congruent than the starting network. This skewed numerical distribution and structured spatial distribution emerge even when boundaries of the topology are eliminated by molding it into a cylinder or torus surface because of the random speed distribution.

The observed emergent behavior and the spatial distributions of speeds and flows are not strange to 
transportation planners; the surprising aspect here is the simplicity and sufficiency of the investment model in replicating observable patterns. The revenue and cost models, which are similar to Cobb-Douglas functions, are realistic simplifications; the revenue in the United States is collected using gas tax and link tolls. It is not implausible to model the cost of maintaining a link as depending largely on the traffic flow, average vehicle speed, and, of course, link length. The investment model used is a link-based decentralized model, while in reality the investment on roads is coordinated by more than one centralized agency and mostly comes in packages applied to multiple links, to exploit economies of scale involved in constructing and maintaining roads. Moreover, these investment packages are spatially heterogeneous in nature; in other words, the rules of transportation investment are not the same for every road or link in a geographical area. Nevertheless, a decentralized investment model is surprisingly sufficient to model the fundamental network dynamics. Such a decentralized model can be justified if it is examined through an induced supply (and induced demand) perspective; transportation planners recommend improving links that are heavily traveled and politicians tend to agree, as it satisfies more constituents. The routes that are proposed for expansion by centrally located decision makers are selected in response to local conditions.

\section{Acknowledgments}

This research is based in part on work supported by the National Science Foundation under Grant 0236396. The assistance of Feng Xie and Lei Zhang is appreciated. All opinions and errors remain those of the authors.

\section{References}

Bak, P. 1996. How Nature Works: The Science of Sef-Organized Criticality. Copernicus (Springer-Verlag), New York.

Barabasi, A.-L., R. Albert. 1999. Emergence of scaling in random networks. Science 286 509-512.
Barabasi, A., R. Albert, H. Jeong. 1999. Scale-free characteristics of random networks: The topology of the World Wide Web. Physica Part A 272 173-187.

Chachra, V., P. M. Ghare, J. M. Moore. 1979. Applications of Graph Theory Algorithms. North Holland, New York.

Christaller, W. 1933. Central Places in Southern Germany. Fischer, Jena, Germany. (English translation by C. W. Baskin. 1966. Prentice Hall, London.)

Fujita, M., P. Krugman, A. J. Venables. 1999. The Spatial Economy: Cities, Regions, and International Trade. MIT Press, Cambridge, MA.

Garrison, W. L., D. F. Marble. 1965. A prolegomenon to the forecasting of transportation development. Technical report, Office of Technical Services, United States Department of Commerce, United States Army Aviation Material Labs, Washington, D.C.

Helbing, D., J. Keltsch, P. Molnár. 1997. Modeling the evolution of human trail systems. Nature 388 47-50.

Hutchinson, B. G. 1974. Principles of Urban Transportation Systems Planning. McGraw-Hill, New York.

Krugman, P. 1996. The Self-Organizing Economy. Blackwell, New York.

Lam, L. 1995. Active Walker models for complex systems. Chaos, Solitons Fractals 6 267-285.

Lam, L., R. Pochy. 1993. Active-walker models: Growth and form in non-equilibrium systems. Comput. Simulation 7 534-541.

Levinson, D., R. Karamalaputi. 2003a. Predicting the construction of new highway links. J. Transportation Statist. 6(2/3) $81-89$.

Levinson, D., R. Karamalaputi. 2003b. Induced supply: A model of highway network expansion at the microscopic level. J. Transport Econom. Policy 37(3) 297-318.

Lösch, A. 1954. The Economics of Locations. Yale University Press, New Haven, CT.

Newell, G. F. 1980. Traffic Flow on Transportation Networks. MIT Press, Cambridge, MA.

Schweitzer, F., L. Schimansky-Geier. 1994. Clustering of active walkers in a two-component system. Physica Part A 206 359-379.

Taaffe, E. J., R. L. Morrill, P. R. Gould. 1963. Transport expansion in underdeveloped countries: A comparative analysis. Geographical Rev. 53(4) 503-529.

Watts, D. J., S. H. Strogatz. 1998. Collective dynamics of "smallworld" networks. Nature 393 440-442.

Yamins, D., S. Rasmussen, D. Fogel. 2003. Growing urban roads. Networks Spatial Econom. 3 69-85. 